\title{
Use of crop sensing technology in crop protection research
}

\author{
D.E. Neill and G.B. Follas
}

Syngenta Crop Protection, Private Bag 92618, Symonds St, Auckland 1150

Corresponding author: dale.neill@syngenta.com

Crop sensing technology is a new tool being rapidly adopted by farmers as a key component of precision agriculture. This technology uses sensors to calculate normalized difference vegetative index (NDVI) by emitting red and near infrared light towards the crop and measuring the crop's reflectance. NDVI is used to evaluate canopy greenness, plant biomass and as an indicator of plant health and vigour. The methodology, relevance and benefits of using this technology in crop protection trials are currently unclear. A handheld Greenseeker ${ }^{\circledR}$ (N-tech Industries, USA) was used to record NDVI on a range of trials from 2008-2011 to establish whether crop sensing could replace visual assessments for disease and enable yield prediction. NDVI readings were compared against other parameters measured in the trials, such as disease scores, green leaf area percentage and yields. In some trials the NDVI followed similar trends to disease, green leaf retention and yields. However, in other cases where clear treatment effects were recorded through visual or yield assessments, there were no differences in NDVI between the treatments. As NDVI can be affected by a number of factors it was concluded that crop sensing technology can be used as an additional objective measurement in conjunction with standard assessment practice but without further investigation cannot replace traditional assessment methods.

\section{Recent developments with methyl isonicotinate, a semiochemical used in thrips pest management}

\author{
D.A.J. Teulon ${ }^{1}$, M.M. Davidson ${ }^{1}$, N.B. Perry ${ }^{1}$, M-C. Nielsen ${ }^{1}$, R.W.H.M. van Tol$^{2}$ and \\ W.J. de Kogel $^{2}$
}

\section{${ }^{1}$ The New Zealand Institute of Plant E Food Research Limited, Private Bag 4704, Christchurch, New Zealand \\ ${ }^{2}$ Plant Research International, Wageningen UR, 6700 AA Wageningen, the Netherlands \\ Corresponding author: David.Teulon@plantandfood.co.nz}

Methyl isonicotinate is one of several recently discovered and patented semiochemicals used in thrips pest management. Methyl isonicotinate is the current active ingredient of LUREM-TR (Koppert Biological Systems) and FROC and THSP (PHEROBANK). These lures are mostly used in Europe but there are plans for the release of these or similar products in Australasia in the near future. Field and glasshouse trapping experiments and wind tunnel and Y-tube olfactometer studies in several countries have shown that methyl isonicotinate has activity against at least 10 thrips species including several important pest species not found in New Zealand. Methyl isonicotinate in LUREM-TR, FROC and THSP is primarily used as a lure with coloured sticky traps for enhanced monitoring of thrips in greenhouses. Recent research indicates that methyl isonicotinate has the potential to be used for other thrips management strategies such as mass trapping, lure and kill, lure and infect and as a behavioural synergist in conjunction with insecticides, in a range of indoor and outdoor crops. 